\title{
Toward a Temporal Theory of Language
}

\author{
RICHARD D. CURETON
}

University of Michigan

During my two years as Sid Greenbaum's colleague at the University of Wisconsin-Milwaukee (1981-1983), one of my prominent memories involves a $\mathrm{Ph} . \mathrm{D}$. exam that he constructed for an English language student in the department. The exam consisted of one question. I forget the exact wording of the question, but it went something like this: What is wrong with The Grammar of Contemporary English; what are its weaknesses and inadequacies? ? $^{1}$ The question is typical of Sid's teaching and scholarship. It does not ask the student what is contained within the limits of our knowledge. It asks the student to explore what lies beyond.

Over the course of my career, Sid's question has been of little concern to me. In fact, I have been mainly concerned with just the opposite-finding ways to present what $G C E / C G E$ does accomplish, rather than what it does not. Each year, I teach one course in modern English grammar, in which I use Sid's A Concise Grammar of Contemporary English, and two courses in the structure of modern English, in which I use Sid's A College Grammar of English (with a coursepack of supplementary notes taken from the more substantial grammars). I also teach various courses in stylistics, and within stylistics, poetics; and in these courses, I use the grammatical system developed in $G C E / C G E$ to teach units on the syntax of prose fiction, drama, and (most intensively) poetry. I also use $G C E / C G E$ in my professional work in stylistics. In the late 70s, I wrote a dissertation on poetic syntax using GCE as my major reference work. During the 80s, I expanded this interest in poetic syntax to prosody, writing a book on verse rhythm, and recently I have become interested in poetics more generally. In this recent work, $G C E / C G E$ has also been a constant companion. In sum, over my career, I have found many useful applications for the grammatical system developed in $G C E / C G E$; therefore, I have had little occasion to reflect on the inadequacies of what it presents.

Lately, however, Sid's question has been more and more on my mind. That is, I have become more concerned with what $G C E / C G E$ lacks rather than with what it provides. Recently, the areas of my pedagogical and scholarly concern (stylistics, poetics, prosody, etc.) have steadily languished, and this languishing is closely connected to the limitations in $G C E / C G E$, in fact, to the limitations in all of our 
contemporary approaches to language. It is this connection between the needs of an adequate stylistics/poetics and the limitations of our current grammars that I will explore here.

Without taking dogmatic stands on controversial issues, GCE/CGE brilliantly synthesizes our two major theoretical approaches to grammatical organization-the formal and the functional. On one hand, syntax consists of a constrained system of forms-a small inventory of word classes (noun, verb, etc.); phrasal and clausal types (noun phrase, intransitive clause, etc.); and realizations of (tense, aspect, voice, mood, number, gender, person, etc.), elaborations of (apposition, coordination, subordination, correlation, etc.), and interconnections and orderings among these phrasal and clausal types (linearization, thematization, focus, pronominalization, ellipsis, etc.).

As $G C E / C G E$ underlines, all grammars must describe these syntactic forms and in doing so, acknowledge their functional autonomy: these forms have no transparent functional motivation, or at least none that we can articulate coherently and convincingly given present knowledge. As any grammar teacher must stress repeatedly, the simple notional definitions of grammatical forms that we inherit from classical times (and that provide the basis of most grammatical pedagogy in the schools) cannot withstand scrutiny. Many nouns do not refer to persons, places, or things. Many adverbs do not modify verbs, adjectives, or other adverbs. Many subjects are not agents. And so forth.

From a functional point of view, the basic organizational features of syntax are mysteries. Why are there just four major word classes: verb, noun, adjective, and adverb? Why is syntax hierarchical and recursive? Why do grammars have phrases as well as clauses, and why are the structures of phrases and clauses distinct? Why are clauses broken into subsections (subject vs. predicate, operator vs. predication, etc.)? Why do grammars need systems of gender and aspect, which mark large subsets of possible nominal and verbal meanings? Why are words less volatile in their structure than phrases, and phrases than clauses? What is a subject, anyway? Our grammars cannot say.

The theoretical primitives of syntactic organization cannot be defined in functional terms. These grammatical forms are just defined by their distribution and collocation in the formal grammatical system of which they are a part. Within linguistic theory, the motivation for grammatical form has been relegated to the mysteries of genetics, neurology, and our species-specific endowment for language (constraints on processing, etc.), matters which have not been connected in any strong way with either the meanings that these forms convey or the social contexts in which these forms are used.

On the other hand, it is also clear that we use grammatical forms in highly constrained and organized ways, and $G C E / C G E$ carefully documents this (functional) use. Most nouns do refer to persons, places, or things. Most verbs do refer 
to actions or states. And so forth. $G C E / C G E$ presents extensive explorations of these grammatical functions, often to high levels of delicacy. In fact, throughout $G C E / C G E$, discussions of semantic function are at least as detailed as discussions of grammatical form. For instance, CGE splits its treatment of verbs into two balanced chapters, a seventy-nine page chapter on verbal forms (93-173) and a sixty-eight page chapter on verbal meanings (173-240). In some of the other chapters (e.g., on adverbials and subordinate clauses), discussions of form and function are combined so intimately that they are impossible to untangle. At least one chapter in CGE is composed primarily of semantic detail: CGE offers only fifteen pages (657-72) that discuss prepositional form followed by thirty-six pages (673-709) that consider prepositional meaning.

As $G C E / C G E$ documents, however, the functional complexity and diversity of grammatical use frustrates any definitional relation between this use and grammatical form. Being cognitively and historically available, grammatical forms can indeed be used to get things done with words, but these uses do not seem to originate from semantically/pragmatically coherent sources, and even so, once placed in circulation, these uses proliferate in ways that are comparable to the proliferation of lexical meanings. For instance, we do indeed use the past tense in English primarily to refer to past time (e.g., I walked the dog yesterday), but we also use the past tense to soften the directness of requests (e.g., Did you want to see me?) and to mark a counterfactual (If I had a nickel, I'd buy a pickle.). These other uses of the past tense have no (literal) relation to time.

The functional descriptions that $G C E / C G E$ claims are not based on any firm theoretical basis either. We know even less about semantics than we do about syntax. While grammars such as $G C E / C G E$ do indeed build up their formal descriptions from certain basic terms and concepts, however unmotivated these basic terms and concepts might be, they do not even attempt this for their functional descriptions; these descriptions are entirely ad hoc. As Chomsky liked to reiterate in the early days of generative grammar, certain areas of linguistics have achieved relatively strong levels of observational adequacy (and even considerable levels of descriptive adequacy), but explanatory adequacy has remained an unrealized dream.

For most practical purposes, this situation is not intolerably disturbing. All of the grammatical phenomena discussed in $G C E / C G E$ receive copious, satisfying comment - on both form and function. The intense formal description makes the grammar a useful resource in documenting usage and adjudicating prescriptive disputes, and the intense functional description makes the grammar useful in many applications-for example, to language awareness, second language learning, dialectology, literary and cultural hermeneutics, and so forth. In fact, outside the realm of pure theory, one might even wonder whether these limitations in the explanatory bases of grammatical description have any serious consequences at all. It would certainly be nice to know in some more principled way what motivates the 
grammatical order in language that we can observe and describe, but, in the end, what would be the value of this knowledge? What aspects of our understanding and appreciation of human nature or culture would be affected by such knowledge, even if it were possible to achieve? Outside the academy, few are even aware that we have no deep understanding of linguistic form and meaning. At lower levels in the schools, and therefore in the society at large, this situation is just ignored. Students are still asked to memorize the ancient "notional" definitions of parts of speech, despite their inadequacy, and more complex grammatical issues are seldom considered.

The major consequence of this explanatory weakness in our linguistic theories, I think, is its effect on our understanding of poetry and the relation between poetic experience and our understanding of the human, both human nature and human culture (including cultural history). As Roman Jakobson liked to remind us, our great poets are our linguistic geniuses. ${ }^{2}$ To an extent that far exceeds our ability consciously to analyze, our great poets achieve a deep intuitive feeling for the intimate relation between the linguistic and the human and therefore a deep feeling for the explanatory bases of language. In fact, as all critics who have thought deeply about this issue have agreed, our great poetry is in some sense about these explanatory bases. Our great poets do not just use language; they thematize the linguistic and therefore give us our best evidence for what language-at the deepest level-is. Jakobson and a few other mavericks aside, this close relation between the linguistic and the poetic has been given much too little attention in contemporary linguistic theory.

The major differences between poetry and other linguistic genres are (1) the unity, concentration, and extent of its use of the materials of language, and (2) its primarily nonreferential, rather than referential, intent. Not being art, most of our uses of language are relatively loose, and even where this use is tight (e.g., in the other language arts: song, prose fiction, and drama), our experience of the selection and arrangement of linguistic forms themselves is not the major point. For example, prose fiction also uses the many resources of language in intensive ways, but largely as a means to another end: to build up fictional worlds of plots, characters, settings, and so on. The central intent of prose fiction is to convey in some maximally effective way what is perceived, felt, done, and thought in those fictional worlds, not to focus on how those worlds are linguistically conveyed. Most prose fiction can survive, with little loss, both paraphrase and transference to another medium (for example, film).

With poetry, however, the opposite is the case. While prose builds worlds from words, poetry builds worlds of words; therefore the fictional worlds that poems convey, if they convey such worlds at all, are more an extension (or, as Amittai Aviram [1994] likes to say, a "telling") of their linguistic form. A novel uses language to tell a story; a poem uses a story to "tell" language. Poems are "telling" 
language, and one of the major things that poems tell us, I think, is just that deep explanation for linguistic organization that our current theories of language, discourse, and culture sorely lack.

The claim that poets "tell" us about language suggests that a principled poetics might give us our best evidence for a principled linguistics, but in itself this claim is still far removed from a solution to the relation between form and function in language. This claim just shifts the burden of explanation to another (equally) troubled field. Our critical theories have yielded revealing accounts of drama and prose fiction, but our accounts of poetry remain sketchy and diffuse. Over the centuries, various principles have been suggested to account for the structure and effect of poetry and poetic language (parallelism, ambiguity, paradox, deviance, foregrounding, iconicity, tension/emotivity, semantic overdetermination, trope, etc.), but taken individually, each of these principles is inadequate, and, taken together, these principles are little more than a chaotic list. At present, we have no principled poetics, either.

A promising way to develop such a principled poetics, I would suggest, is to take seriously the common claim that poetry is essentially a musical, and therefore rhythmic, art - the claim that the basic concern of poetry is with time and human inwardness rather than space and human outwardness. In this approach to poetry, the human forms that poems present are temporal forms and, to borrow Aviram's terms again, various "tellings" of those forms into other media and modes. Given that poetic worlds are largely worlds of words, this claims that the myriad forms of both language itself and all of the other resources of the language arts (rhetoric, discourse, etc.)-what organizes their inventories of elements, levels of structure, arrays of functions, and the like-are temporal, too. How this can be so is a long story, one that exceeds by far the small space I am allocated here, but with the space I have, I can sketch the basic contours of this claim. ${ }^{3}$

The first step in this temporal approach to the language arts is to clarify the nature of human time. In the normal case, we think of human time as clock time, but this is a mistake. Clock times are not human times; they are externally imposed. In essence, they are ways of coordinating space, a fourth dimension of space added as a further control to the standard three. Human times are rhythmically constructed; they have little or nothing to do with clock time. As rhythms go, so goes time.

There are four types of human time, four types of human rhythm. While we do not know these things for sure, my guess is that each of these times/rhythms is associated with a major part of the brain, which is also quadratic in its form. We have four brains: a reptilian/hind brain, a mammalian/mid-brain, a left cortex, and a right. Each of these human rhythms has a relatively distinct form and therefore a relatively distinct collection of representational powers. Because these rhythms reflect the evolutionary history of the brain, features of these representational 
powers are also dialectically arrayed: each additional rhythm developed to overcome the representational limitations of its predecessor. As most theories of the human point up, human sensibility is essentially dialectical in form.

These four different rhythms can be recognized in our experience of any temporal medium, but they are perhaps most evident in music, the most purely temporal art. Musicians call these four rhythms meter, grouping, prolongation, and theme. ${ }^{4}$

Meter is a beating: 1-2-3-4. It is essentially a response to tactile weight; it is very subjective (or representationally poor); it is primarily physical/gestural in source; and, while being performed, it strongly invites participation (we move with the rhythmic stimulus). Structurally, it is declining in its contours of prominence; it is relatively local in its scope of operation; it tends toward rigidly repetitive, binary patterns; and it is essentially an ordering of identical events: beats of the same strength are the same in other respects, too. As a result of these features, meter constructs (what we might call) "cyclical" time.

Grouping is more flexible, divisive, and inclusive. It phrases a stimulus into parts (and those parts into parts), each of which has some peak of prominence. The essential motion of grouping is inward: it repetitively groups structurally weak events around structurally strong events, ordering the result into a coherent hierarchy. In constructing this hierarchy, grouping is still fairly subjective, but it is more responsive to a rhythmic stimulus than meter: it searches out differences among sames. It is more proportional than repetitive, more concerned with global structural dependencies than with local tactile weights, and more sensitive to climaxes than to onsets (and therefore more concerned with middles than with beginnings). While meter winds down and therefore falls in prominence, grouping prefers to rise, crescendo. In music, grouping is closely associated with harmony. In its spatial analogues, grouping resembles architecture. As a result of these features, grouping constructs (what we might call) "centroidal" time.

Prolongation is more prospective and asymmetrical. It represents progress to and from structural goals and therefore generates complex feelings of anticipation, arrival, departure, and structural connection/continuity. If grouping keeps track of differences among sames, prolongation tracks sames among differences. It evaluates passing events for their causal connections, viewing events according to some imposed perspective and, in so doing, evaluating the functional cooperation of events within that perspective. If meter is concerned with the onsets of events and grouping with their middles, prolongation is concerned with their ends-with closure, termination, structural satisfaction/complementation. In music, prolongation is strongly related to melody. As a result of these features, prolongation constructs (what we might call) "linear" time.

Theme is more volatile and discontinuous. It tracks contextual differences among events that share some family resemblance. While the other rhythms are 
TABLE 1

The Temporal Paradigm

\begin{tabular}{|c|c|c|c|c|}
\hline Temporal Features & Cyclical & Centroidal & Linear & Relative \\
\hline Event-event relation & Similarity & $\begin{array}{l}\text { Difference-in- } \\
\text { similarity }\end{array}$ & $\begin{array}{l}\text { Similarity-in- } \\
\text { difference }\end{array}$ & Difference \\
\hline Temporal figure & $\begin{array}{l}\text { Occurrence } \\
\text { Repetition } \\
\text { Succession }\end{array}$ & $\begin{array}{l}\text { Correspondence } \\
\text { Prominence } \\
\text { Proportion }\end{array}$ & $\begin{array}{l}\text { Transition } \\
\text { Direction } \\
\text { Implication }\end{array}$ & $\begin{array}{l}\text { Connection } \\
\text { Distinction } \\
\text { Simultaneously }\end{array}$ \\
\hline $\begin{array}{l}\text { Subject-subject } \\
\text { relation }\end{array}$ & Participation & Obligation & Cooperation & Freedom \\
\hline $\begin{array}{l}\text { Subject-event } \\
\text { relation }\end{array}$ & Subjective & $\begin{array}{l}\text { Objective-in- } \\
\text { subjective }\end{array}$ & $\begin{array}{l}\text { Subjective-in- } \\
\text { objective }\end{array}$ & Objective \\
\hline Semiotic relation & Sign & Icon & Index & Symbol \\
\hline Cognitive process & $\begin{array}{l}\text { Reaction } \\
\text { Passive }\end{array}$ & $\begin{array}{l}\text { Affection } \\
\text { Reciprocal }\end{array}$ & $\begin{array}{l}\text { Expression } \\
\text { Active }\end{array}$ & $\begin{array}{l}\text { Creation } \\
\text { Improvisatory }\end{array}$ \\
\hline Clock time & Past & Present & Future & Relative \\
\hline Relational scope & Proximate & Local & Regional & Global \\
\hline Event position & Initial & Medial & Final & Peripheral \\
\hline $\begin{array}{l}\text { Curve of energy/ } \\
\text { stability }\end{array}$ & Fall & Rise-fall & Fall-rise & Rise \\
\hline Structural volatility & Fixed & Constrained & Volatile & Free \\
\hline
\end{tabular}

strongly directed, theme is adirectional or multidirectional; it establishes simultaneous connections among events throughout a medium, weighing each event according to its differential relation to many others. The basic structural figure in theme is novelty/play. Structurally, it is unconstrained/free. When elaborated in full, thematic connections are strongly representative and therefore approximate an objective representation. If the temporal experience is complex, its thematic texture will be unique. Given these features, theme constructs (what we might call) "relative" time.

These features of our four human times-cyclical, centroidal, linear, and relative-can be organized into a featural matrix that represents their dialectical relations on a range of general parameters. I like to call this featural matrix "the temporal paradigm."

The basic claim I would like to make is that this temporal paradigm can provide an informing substructure of a principled poetics (and, therefore, a principled linguistics). The featural composition of each of these times, I would claim, forms a distinct structural "complexion" that can be discerned throughout the disparate materials that constitute the language arts and their contexts of use. In fact, these structural complexions seem to have an even broader scope. They seem to represent four natural temporal logics that occur throughout the biological world-both 
human and non. As the common products of evolutionary selection, all biological forms share certain common structural traits. The four featural complexions in the temporal paradigm represent what might be some of the most general of these resemblances. From this perspective, these featural complexions might be viewed as a type of evolutionary sediment, highly valued organizational forms that, from the pressures of chance and effectiveness, have been selected again and again by evolutionary history, with human neural anatomy, and therefore human sensibility and human language, being instances of this selection.

The most basic fact about the temporal paradigm is its quadratic organization. This quadratic organization claims that, in all human experience, domains of activity tend to fracture into fours according to our four temporal capabilities. As an activity gets more complex and in itself evolves a system of forms, this quadratic organization will be reproduced again and again, in fractal arrays. Fours will break into fours, which will break into fours, and so forth, to some limit of evolutionary delicacy. While I certainly do not have time to explicate all of these correlations here, these myriad embodiments of the four temporalities can also be represented in paradigmatic form. I like to call this "the poetic paradigm."s

With respect to human sensibility, the claims of this poetic paradigm converge with the traditional view that the major human faculties are sense, feeling, will, and thought/memory. Culturally, these claims converge with a quadratic analysis of the cultural history of the West: Ancient, Medieval/Renaissance, Romantic/Nineteenth century, and Modern/Postmodern. Linguistically, these claims converge with a quadratic theory of levels of linguistic organization: paralanguage, prosody, syntax, and semantics. Rhetorically, these claims converge with a quadratic theory of the "master" tropes: metaphor, synecdoche, metonymy, and irony. Critically, these claims converge with Northrop Frye's quadratic theory of the literary genres and modes of emplotment: epic, lyric, narrative, and dramatic; romance, comedy, tragedy, and satire. Philosophically, these claims converge with Stephen Pepper's quadratic analysis of metaphysical systems: formism, organicism, mechanism, and contextualism. Semantically, these claims re-invoke the Aristotelian categories of quantity, quality, relation, and manner. And so forth. ${ }^{6}$

Much work will be needed to work out exactly what motivates these quadratic arrays in each case and exactly how these arrays relate to one another within language as a whole, but even at this early, speculative stage, the power of the temporal and poetic paradigms to explain many of the inventories of forms claimed by grammars such as $G C E / C G E$ is evident. For instance, in the light of this quadratic theory, the existence of many of the quadratic arrays claimed by $G C E / C G E$ fall out as a matter of course-the four major levels of grammatical organization (word, phrase, clause, sentence), the four major word classes (noun, adjective, verb, and adverb), the four basic sentence patterns (declarative, exclamative, imperative, interrogative), the four basic moods (indicative, subjunctive, imperative, infinitive), 
the four basic elements of the clause (subject, verb, complement, adverbial), the four major subcategories of adverbials (adjunct, subjunct, conjunct, and disjunct), the four basic functions marked in the verb phrase (voice, aspect, modality, and tense), the four basic functions marked in the noun phrase (number, gender, case, and person), the four basic tenses (past, non-past, future, relative), the four basic voices (passive, middle, active, causative), the four basic aspects (perfective, imperfective, progressive, perfect), the four basic sorts of reference (generic, indefinite, definite, proper). And so forth.

While I do not have space here to explicate these motivations in full, the features of the four temporalities presented in the temporal paradigm also go a long way toward motivating the relative positioning of elements in these quadratures with respect to the four temporalities. For instance, the features of the centroidal temporality connect conjunction, paradox, prosody, phrases, adjectives, modification, subordination, hyponymy, aspect, and gender on the basis of their concern for prominent differences among sames (and therefore essential parts/subsets within containing wholes). They link these forms to rhetorical schemes such as chiasmus and assonance in terms of their concern for structural middles versus structural peripheries. They link these forms to the imperfective, the present tense, subjuncts, clitic phrases, and the first person by their concern for local relations. They link these forms to exclamatives and rise-fall intonation by their wavelike motion and concern with emotion/affection. They link these forms to things like iconicity, parallelism, simile, allegory, the pentameter, and the stanza by their concern for correspondence and proportion. And so forth. As in preference rules systems (or, more recently, optimality theory), the relations between particular structures and one of the four temporalities are often determined by a convergence of several features in the temporal paradigm.

A particularly striking feature of the fractal organization of this theory is its ability to account for larger catalogs of forms, catalogues that are not multiples of four, but something else. For instance, $C G E$ (721) claims that there are exactly seven basic clause patterns in English: SV, SVO, SVC, SVA, SVOO, SVOC, and SVOA. $C G E$ presents these patterns in no particular order and does not relate them in any principled way to other structures in the grammar. Organizing these seven patterns into a quadratic fractal, however, yields the following elegant organization:

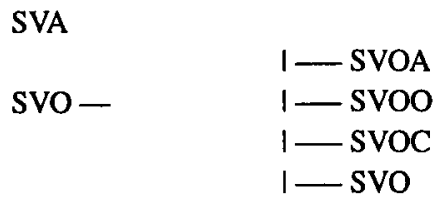

SVC 
This fractal representation also helps to motivate the asymmetrical elaboration within this paradigm. Within language as a whole, syntax is a reflex of linear time. Among levels of syntactic structure, the clause is also a reflex of linear time. And among basic clause patterns, transitives are a reflex of linear time, too. Therefore, we might expect clausal structures to offer more transitive options than, say, intransitives or copulars. In fact, given that syntax is linear, this theory would predict that linear processes would appear more elaborated throughout the grammar-and this does indeed seem to be the case. In general there are more types of clauses than phrases, more cases than genders, more modalities than aspects, and so on.

This quadratic theory of human temporality is especially useful in motivating the relationships among the myriad prosodic, linguistic, rhetorical, and cultural forms that occur simultaneously in both individual poems and in our major poetic styles (and, in turn, such stylistic convergences stand as evidence for the grammatical claims of the temporal and poetic paradigms). The major difficulty with our poetic theories has been their inability to connect such converging and co-occurring stylistic features with the major purposes of poetry as an art. The theory of temporality I am suggesting here claims that the central intent of poetry is always a temporal dialectic. Depending on its historical positioning, a poem will front one or another of these temporalities and then consider the difficulties this unnatural temporal dominance entails for a full human existence, "telling" out this temporal dilemma, first, into rhythmic forms themselves, and then into those linguistic, rhetorical, and referential structures that "tell" the temporalities that these rhythmic forms invoke and represent.

I do not have space to present a complex example here, but consider a simple instance: the opening six lines of Whitman's "Song of the Broad-Ax."”

Weapon shapely, naked, wan,

Head from the mother's bowels drawn,

Wooded flesh and metal bone,

Limb only one and lip only one,

Gray-blue leaf by red-heat grown,

Helve produced from a little seed sown,

Resting the grass amid and upon,

To be lean'd and to lean on.

In the "Song of the Broad-Axe" as a whole, Whitman uses the central figure of the broad-axe to celebrate "strong shapes and attributes of strong shapes," a theme that he extends in his characteristic way to include the strength of the body and physical work and many other dimensions of American culture that relate to these: the strong American personality, the strength of our democratic politics, the might of our industrial production, the breadth and depth of our historical, geographical, and ethnographical diversity, among others. 
To do this, he opens the poem with six lines that bend all of the resources of language to a cyclical temporality, the temporality of the body. The basis for this style, it seems evident, is Anglo-Saxon verse, the style that most closely approximates his purposes, but he modifies this traditional form so that it even more purely invokes the physical/cyclical.

These six visual lines break into eight metrical lines that pound out their binary meter to seven levels. By strongly controlling the number of syllables and stresses between tactical beats, Whitman also strengthens the feeling that the meter is dominating the language. The final line promotes its projectional beats (the two instances of to) in an awkward fashion and strongly syncopates lean. This syllabism energizes the meter in places where superfluous syllables and stresses collect or expected syllables are lost (e.g., the consistently acephalous lines, the two instances of only in line three, and in lines six and seven).

Like the Anglo-Saxon, Whitman supports this dominating meter with the sonic equivalent of meter, alliteration, among tactical syllables within lines (weapon-wan, limb-lip, gray-grown, seed-sown, etc.). But again, he strengthens this cyclical sound by alliterating repeated words, unstressed syllables, stressed syllables off the beat, and syllables across lines as well (e.g., wooded-one-one, gray-grown-grass, headheat-helve, mother's-metal-amid, shapely-produced-upon, limb-lip-leaf-littlelean'd-lean, produced-drawn, etc.). In an unusual gesture, both for him and for this verse form, Whitman also rhymes his lines, but again in support of a cyclical temporality, he blurs these rhymes so that they suggest just one, eightfold repetition: wan, drawn, bone, one, grown, sown, upon, on.

Whitman also supports this meter with other prosodic and rhythmic structures. For instance, most words of more than one syllable have falling stress contours (weapon, shapely, naked, mother's, wooded, metal, only, only, gray-blue, red-heat, little, resting). Terminal tones on intonational contours are also predominantly falling. (The passage is not itself a sentence, but the parts of the sentence that are presented are declarative in structure and function.) Following meter as well, grouping structures often fall (e.g., limb only one and lip only one) and prolongation often extends (e.g., lines one and two, which both present nominal heads-weapon, head-and then modify them-shapely, naked, wan; from the mother's bowels drawn).

Whitman also uses a cyclical syntax and morphology. He uses compounds (gray-blue, red-heat), apposition of various sorts (shapely, naked, wan; weapon ..., head ..., flesh . ., limb ..., etc.), nouns (weapon, head, flesh, bone, etc.), generic reference (weapon, head, flesh, etc.), passives (drawn, grown, produced, to be lean'd), intransitives (resting, lean), material and nominal modifiers (wooded, metal), conjunction (limb ... and lip, amid and upon, to be lean'd and to lean on), ellipsis (throughout), color modifiers (wan, gray-blue, etc.), limiter subjuncts (only, only), and numerals (one, one). 
He also supports the meter with a cyclical rhetoric: metaphor (the mother's bowels, wooded flesh, metal bone), fronting inversions (the grass amid and upon), tight parallelism (throughout), and exact repetition (only one, only one, etc.).

Finally, almost all of the references in these lines are cultural correlates of the cyclical. The poem makes explicit reference to origins (drawn, grown, sown), unity (one, one), hunting and gathering (the broad-axe itself), kinship (mother's), nature (leaf, seed, grass), the body (naked, bowels, head, flesh, bone, limb, lip), the senses (gray-blue, red-heat, wan), touch (red-heat, resting, lean on), and the military (weapon).

This is a very simple instance of the explanatory power of the temporal approach to poetry I am suggesting. But even here, the results are significant. In the light of this theory, the convergence of just these stylistic features in just this context is no accident. The temporal and poetic paradigms explain in a principled manner why this occurs. Poetry "tells" time; the temporal and poetic paradigms give us detailed access to the rhythmic sources of these "tellings."

The temporal theory of language that I am suggesting is a major hypothesis about the nature of language, and as with all such large hypotheses, it will take a long time to work out its claims in full. However, if this suggestion can be worked into a full account of language, it will constitute a striking intervention in linguistic theory. ${ }^{8}$

First, this temporal theory of language is interventionist in its shift in cognitive/ metaphysical mode from space to time. All of our theories of language have been oriented toward space: Aristotelian logic, classical rhetoric, communication theory (e.g., Jakobson's anatomy of speech functions: phatic, conative, expressive, referential, etc.), Skinner's behavioralism, Halliday's social semiotics (textual, interpersonal, ideational, etc.), Langacker's cognitive grammar (image-schemas, etc.), speech act theory (Bakhtin, Austin, Searle), and so forth. Even our most dogmatically formalistic theories (e.g., Bloomfieldian structuralism and Chomskian minimalism) have never suggested that language is essentially temporal.

Second, this temporal theory of language is interventionist in its suggestion that the core structures in language are rigorously fractal. Many have claimed that language is structural, rule-governed, hierarchical, recursive, modular, rankshifting, and many other things that are closely related to fractal form. But to my knowledge, no one has ever suggested that-like clouds and mountain rangeslanguage is also "generated" by preserving the results of an evolutionary iteration that folds a simple structure self-reflexively within itself to minute levels of delicacy. The most striking result of fractal form is a tight family resemblance among disparate units, structures, events, and processes within the containing whole. It is just these family resemblances, I maintain, that poets "know" and poems "tell." 
Third, this temporal theory of language is interventionist in providing a rich linguistic formalism. The major difficulty with our formal theories of language has been their poverty. More than anything else, it is this poverty that makes theorists reject formalistic approaches to language for some more inclusive functionalism. The temporal theory of language that I am suggesting is both formal and maximal.

Fourth, this temporal theory of language is interventionist in its emphasis on similarities. As linguists have developed more rigorous theories of language, they have tended to compartmentalize linguistic phenomena, underlining their differences. As a result of this emphasis on difference, our more inclusive theories of language tend to be unmanageably diffuse. It has been this unnatural valorization of difference that has rendered our linguistic theories of so little use in literary study (stylistics, poetics, etc.). While our linguistic theories can describe the language of poems in great detail, those descriptions, with their obtuse valorization of linguistic difference, are (what I like to call) "analytical heaps," and such "heaps" have little resemblance to art. By stressing similarities rather than differences, the temporal theory of language that I am suggesting overcomes these difficulties. It makes possible a tightly integrated linguistics. The temporal and poetic paradigms provide the bases for this integration.

Fifth, the temporal theory of language that I am suggesting is interventionist in that it provides an independently motivated and therefore truly empirical formalism. To this point, formalist theories of language have had to rely heavily on various nonempirical evaluative assumptions (economy, simplicity, etc.), and even so, the application of these nonempirical assumptions has been wildly uneven. The temporal theory of language that I am suggesting can dispense with these nonempirical assumptions. In this temporal theory, a linguistic description is well motivated to the extent that it can be derived from the temporal paradigm; the temporal paradigm catalogs and interrelates the most prominent qualities of rhythmic form; and these qualities of rhythmic form can be experienced directly in many ways-for example, in the rhythms of music, dance, poetry, and so forth.

Sixth, the temporal theory of language that I am suggesting is interventionist in its claim that the core structures in language are the product of an evolutionary process. The very possibility of organizing the prominent features of human rhythms into the temporal paradigm results from viewing each subsequent rhythmic "component" as a "solution" to the inherent limitations in its rhythmic predecessormeter leads to grouping, which leads to prolongation, which leads to theme. This fourfold evolution then bends back on itself into an infinite loop (i.e., the relative leads back to the cyclical, and the evolution repeats). This Viconian "recurso" is the basis of the coherence of the temporal paradigm, which moves from similarity to difference (and then back to similarity), repetition to distinction (and then back to repetition), succession to simultaneity (and then back to succession), subjective to 
objective (and then back to subjective), fall to rise (and then back to fall), fixed to free (and then back to fixed), reaction to creation (and then back to reaction), passivity to improvisation (and then back to passivity), proximate to global (and then back to proximate), and so forth. This temporal theory of language claims that the underlying "logic" of language is biological; it is not mathematical, sociological, semiotic, or whatever.

Seventh, this temporal theory of language is interventionist in that it creates a deeply humanistic linguistics. The explanatory bases of language, this theory maintains, are also the explanatory bases of the other major products of mind-our senses, our psychological faculties, our social institutions and their historical development, and so forth. Most dramatically, this theory claims that the explanatory bases of language are also at the basis of the arts (music, dance, poetry, etc.) and, in fact, are best observed there. One of the saddest chapters in the history of linguistics in this century has been the widening gap between the methods and intentions of linguistic versus literary studies, and between both of these and study of mind and culture more generally. The temporal theory of language I am suggesting re-humanizes the linguistic.

Eighth, this temporal theory of language is interventionist in its inclusiveness, and therefore in its expansive and unifying effect on the study of language proper. Despite the admirable attempts by recent functional theories of language to become more inclusive (e.g., treating semantics and pragmatics as well as grammar and phonology, prosody and paralanguage as well as syntax), to preserve the coherence of their functional approaches, all such theories exclude certain central linguistic phenomena from their consideration. The most glaring instance of this, certainly, is the exclusion of rhetorical tropes and schemes from most contemporary theories of language, a gesture that divorces the modern study of language from the major focus of the study of language in ancient times. The temporal theory of language I am suggesting reunites the language arts.

Finally, the temporal theory of language that I am suggesting does not just reunite and humanize linguistics and therefore make it sufficiently inclusive to consider the language arts, it repositions the language arts back to the center of linguistic theory. It reinvokes the ancient wisdom that our poets are our first and best linguists, and therefore if we are to arrive at a deep understanding of language, the language of poetry should be our first and best concern. The pretentious (and often empty) scientism of much of modern linguistics has had the result of alienating from our most intense and serious explorations of language just those who are most intimately familiar with this central concern. Within our major universities, almost no linguistics departments include scholars whose basic concern is to explore the language arts, and in linguistic curricula, courses that examine the relation between language and art are almost nonexistent. From the point of view of most linguistic theories, poetic uses of language have been considered little more than precious 
and peripheral affairs, as though the art were a minor concern in human life and therefore of minor import in the organization of human cognition, sensibility, and therefore language.

The temporal theory of language that I am suggesting stands this attitude on its head. If language is indeed a fractal elaboration of our human sensibilities, as this theory claims, a major result is that language becomes essentially beautiful; therefore, the "logic of linguistic theory," if it is to follow what language is rather than some set of external methodological constraints, must follow the contours of the beautiful as well. The most productive way to arrive at a deeper understanding of language, this theory implies, is to explore the role of beauty in human evolution, sensibility, psychology, cultural formation, and cultural history.

\section{Notes}

1. The revised and expanded version of GCE, The Comprehensive Grammar of English (CGE), appeared in 1985. These grammars are different in many ways, but the general issues that I am considering apply to both.

2. Jakobson's best work in poetics has now been conveniently collected in Jakobson (1987).

3. For a more detailed presentation and exemplification of the argument presented here, see Cureton (in press).

4. For a longer discussion of the first three of these rhythmic components in both poetry and music, see Cureton (1992). For shorter summaries of these matters, see Cureton (1993, 1994a, 1994b). For the best one-volume discussions of musical rhythm, see Lerdahl and Jackendoff (1983) on classical tonal music and Kramer (1988) on more contemporary forms.

5. For a lack of space, I do not print the poetic paradigm here. I think that my argument and its applications to language are understandable without it. My major claims here refer directly to the temporal paradigm. For a printing of the poetic paradigm, see Cureton (in press).

6. See Frye $(1957,1963,1981,1990)$ and Pepper (1942). The intellectual sources of the poetic paradigm are large and cannot be cited in full here. For a succinct discussion of such quadratic organization in culture and history, see Thompson (1971). For an extensive development of Frye, see White $(1973,1978,1987)$. For some of the biological and psychological bases of this quadratic organization, see Anderson (1995), Donald (1991), and MacLean (1973). See Ong (1982) for the features of oral societies, Lovejoy (1936) and Tillyard (1942) for features of Medieval and Renaissance culture, Foucault (1973) and McLuhan (1962) on features of nineteenth-century culture, and Jameson (1991) for features of postmodernism. For a history of English poetry that uses these psychological bases, see Easthope (1983). 
7. Whitman $(1973,184)$.

8. The linguistic theory that resembles a temporal approach to language most closely is Kenneth Pike's tagmemics, especially his analysis of grammatical phenomena into four-cell tagmems: unit, slot, role, and relation. For some work on tagmemics, see Pike $(1959,1967,1982)$ and Pike and Pike $(1982,1983)$.

9. This centering of time, beauty, and the arts in biological evolution and cultural activity resembles Frederick Turner's "natural classicism" and its sources in the work of J. T. Fraser and the Society for the Study of Time. See Turner $(1991,1992)$ and Fraser (1966, 1975), and Fraser et al. (1972-89).

\section{References}

Anderson, Clifford. 1995. The Stages of Life. New York: Atlantic Monthly Press. Aviram, Amittai F. 1994. Telling Rhythm. Ann Arbor: University of Michigan Press. Cureton, Richard D. 1992. Rhythmic Phrasing in English Verse. London: Longman.

. 1993. The Auditory Imagination and the Music of Poetry. In Literary Stylistic Studies of Modern Poetry, edited by Peter Verdonk, 68-86. London: Routledge.

- 1994a. Rhythm and Verse Study. Language \& Literature 3:105-24. 1994b. Rhythmic Cognition and Linguistic Rhythm. Journal of Literary Semantics 23:220-32.

- in press. Linguistics, Stylistics, and Poetics. Language \& Literature.

Donald, Merlin. 1991. Origins of the Modern Mind. Cambridge, MA: Harvard University Press.

Easthope, Anthony. 1983. Poetry as Discourse. London: Methuen. Foucault, Michel. 1973. The Order of Things. New York: Random House.

Fraser, J. T., ed. 1966. The Voices of Time. Amherst: University of Massachusetts Press.

- 1975. Of Time, Passion, and Knowledge. Princeton: Princeton University Press.

Fraser, J. T., et al., eds. 1972-89. The Study of Time I-VI. New York: Springer Verlag. Frye, Northrop. 1957. Anatomy of Criticism. Princeton, NJ: Princeton University Press.

- 1963. The Well-Tempered Critic. Bloomington: Indiana University Press.

- 1981. The Great Code. New York: Harcourt Brace Jovanovich.

- 1990. Words with Power. New York: Harcourt Brace Jovanovich. Greenbaum, Sidney. 1989. A College Grammar of English. London: Longman. Jakobson, Roman. 1987. Language in Literature, edited by Krystyna Pomorska and Stephen Rudy. Cambridge, MA: Harvard University Press. Jameson, Fredric. 1991. Postmodernism. Durham, NC: Duke University Press. Kramer, Jonathan D. 1988. The Time of Music. New York: Schirmer. 
Lerdahl, Fred, and Ray Jackendoff. 1983. A Generative Theory of Tonal Music. Cambridge: MIT Press.

Lovejoy, Arthur O. 1936. The Great Chain of Being. Cambridge, MA: Harvard University Press.

MacLean, Paul. 1973. A Triune Concept of Brain and Behavior. Toronto, Canada: University of Toronto Press.

McLuhan, Marshall. 1962. The Gutenberg Galaxy. Toronto, Canada: University of Toronto Press.

Ong, Walter. 1982. Orality and Literacy. New York: Methuen.

Pepper, Stephen. 1942. World Hypotheses. Berkeley: University of California Press. Pike, K. 1959. Language as Particle, Wave, and Field. The Texas Quarterly 2:37-54. 1967. Language in Relation to a Unified Theory of the Structure of Human Behavior. $2 \mathrm{~d}$ ed. The Hague, the Netherlands: Mouton. 1982. Linguistic Concepts. Lincoln: University of Nebraska Press.

Pike, K., and Pike, E. 1982. Grammatical Analysis. Arlington, TX: The Summer Institute of Linguistics.

1983. Text and Tagmeme. Norwood, NJ: Ablex.

Quirk, Randolph, and Greenbaum, Sidney. 1973. A Concise Grammar of Contemporary English. New York: Harcourt, Brace, Jovanovich.

Quirk, Randolph, et al. 1972. A Grammar of Contemporary English. London: Longman.

Longman.

Thompson, William Irwin. 1971. At the Edge of History. New York: Harper \& Row. Tillyard, E. M. W. 1942. The Elizabethan World Picture. New York: Random House. Turner, Frederick. 1991. Beauty. Charlottesville: University of Virginia Press. 1992. Natural Classicism. Charlottesville: University of Virginia Press.

White, Hayden. 1973. Metahistory. Baltimore: Johns Hopkins University Press. 1978. Tropics of Discourse. Baltimore: Johns Hopkins University Press. 1987. The Content of the Form. Baltimore: Johns Hopkins University Press.

Whitman, Walt. 1973. Leaves of Grass, edited by Sculley Bradley and Harold Blodgett. New York: Norton. 